\title{
Evaluation of Adequate Time for Intravenous Cannulation in Children during Sevoflurane Induction
}

\author{
Ben Ali.M*, Stambouli L, Hafi F, Ben Salem F, Gahbiche. M \\ Department of Anesthesiology and Intensive care FB Hospital Monastir, Tunisia
}

*Corresponding Author: Ben Ali.M, Department of Anesthesiology and Intensive care FB Hospital Monastir, Tunisia

\begin{abstract}
Background: The early placement of an intravenous (iv) cannulation in children anesthetized with halothane has been shown safe and acceptable compared with later placement. However, there's not well known with sevoflurane use [2].
\end{abstract}

Aim: The aim of our study is to determine whether one should make iv attempts during the early induction period (at 60seconds) or lately (90 or 120 s).

Methods: We conducted a prospective randomized study, after obtaining Institutional review board approval, which didn't require written parental consent. The Trial registration was (Protocol ID : 09250586 ; Clinical trial.gov ID : NCT03851692). Children age 1-12 years, undergoing elective general anesthesia via an inhalation induction were randomized to one of three groups of 30 patients each one, for iv placement, either 60s (group E), 90 or $120 \mathrm{~s}$ (group L) following loss of lid reflex.

Results: The study groups were comparable in term of age $(p=0.48)$, Sex, weight and ASA $(p=0.33)$. Movement on iv placement happened in 12 cases of group E but any case in group L $(p<0.0001)$. There was no case of laryngospasm in each group. The iv placement was better at first attempt in 29 patients of group $L(120 s)$ vs 27 others of group L (90s) and 22cases of group E with significant difference $(p=0.02)$.

Conclusion: We recommend waiting unless 90 or better 120 s following the loss of lid reflex before attempting iv placement in children receiving an sevoflurane inhalation.

Keywords: Child; general anesthesia; induction of anesthesia; sevoflurane inhalation; intravenous cannulation.

\section{INTRODUCTION}

Sevoflurane has been in clinical use for inhalation anesthesia for more than 20 years and has been tested in numerous studies [1]. Sevoflurane has been described as the agent of choice for mask induction in children due to itslack of airway irritation, hemodynamic characteristics, and lower pungency [2]. Inhalation induction (IND) with sevoflurane is the preferred method for anesthetizing children in pediatric anesthesia. The ideal time for intravenous (IV) cannulation following inhalational induction in children is debatable. Its rapid halothane exposure has been reported to be better in terms of safety with fewer side effects than its late location. However, its early exposure to sevoflurane is poorly studied in the literature [3]. The administration of high concentrations from the start to 7 or $8 \%$ allows a faster and often preferred IND in children.

\subsection{Objective}

To determine the appropriate delay, early (60s) or late $(90$ or $120 \mathrm{sec}$ ), in VVP after cerebral reflex loss with sevoflurane in children, with the least adverse effects.

\section{MAterial AND Methods}

We conducted a prospective, randomized, double-blind study, after obtaining Institutional review board approval from the Ethics Committee, which didn't require written parental consent. Date of approval: 27 juilliet 2016. It was prospectively registered with the Clinical trial.gov. The Trial registration was (Protocol ID : 0925-0586 ; Clinical trial.gov ID : NCT03851692). Our study carried out in 6month (from 01 Aout 2016-to 31 Janvier 2017), that includes all children with ASA physical status I, II, aged 1 to 12 , proposed for ambulatory surgery, under general anesthesia. 
The criteria for non-inclusion are: Age $<1$ year or> 12 years, non-ambulatory surgery, contraindication IND sevoflurane. The children are randomized into 3 groups (computerized randomization) before the anesthetic IND according to the time of insertion of the venous cannulation is 60,90 or 120 seconds after the loss of the ciliary reflex. No premedication previously instituted in patients. All children are monitored by standard monitoring. Induction (IND) at $8 \%$ sevoflurane. Spontaneous ventilation is then maintained in all patients throughout the IND with manual ventilatory support as needed. Children randomized to the early group will have an attempt to apply the venous approach $60 \mathrm{~s}$ after loss of the ciliary reflex while for the late group, the VVP is performed at $90 \mathrm{~s}$ (late group 90s) or $120 \mathrm{~s}$ (late group 120s) after loss of ciliary reflex. At the time of insertion of VVP, the presence of movements and the occurrence of laryngospasm are noted by an independent observer and are recorded for each study group. Judgment criteria were in primary outcome measure movement on iv placement and incidence of laryngospasm,

\begin{tabular}{|l|l|l|l|l|}
\hline & $\begin{array}{l}\text { Early Group G1 (60s) } \\
\mathbf{n = 3 0}\end{array}$ & $\begin{array}{l}\text { Late Group G2 (90s) } \\
\mathbf{n = 3 0}\end{array}$ & $\begin{array}{l}\text { Late Group G3 } \\
(\mathbf{1 2 0 s}) \mathbf{n = 3 0}\end{array}$ & P value \\
\hline Age (years) & $3,11 \pm 2,09$ & $3,75 \pm 2,17$ & $3,26 \pm 1,9$ & 0.485 \\
\hline Weight(Kg) & $14,17 \pm 4,51$ & $16,73 \pm 7,69$ & $17 \pm 6,05$ & 0.9 \\
\hline Sex (M/F) & $25 / 5$ & $26 / 4$ & $25 / 5$ & 0.9 \\
\hline
\end{tabular}

Movement on iv placement happened in 12 cases of group $\mathrm{E}$ but any case in group $\mathrm{L}$ $(p<0.0001)$. Older children in group $\mathrm{E}$ were more likely to have significant movement. There was no case of laryngospasm in each group. The iv placement was easy, at first attempt in 22

Table2: Data and incidents in 3 groups

\begin{tabular}{|l|l|l|l|l|}
\hline & $\begin{array}{l}\text { Early Group } \\
\text { G1 (60s) } \mathbf{n = 3 0}\end{array}$ & $\begin{array}{l}\text { Late Group } \\
\text { G2 (90s) } \mathbf{n = 3 0}\end{array}$ & $\begin{array}{l}\text { Late Group } \\
\text { G3 (120s) } \mathbf{n = 3 0}\end{array}$ & P value \\
\hline Movement on IV placement & 12 & 0 & 0 & $<0.0001$ \\
\hline Laryngospasm & 0 & 0 & 0 & \\
\hline $\begin{array}{l}\text { Nb of Attempts : } \\
\text { 1st attempt }\end{array}$ 2nd attempt & 22 & 27 & 29 & \\
& 8 & 3 & 1 & 0.024 \\
\hline
\end{tabular}

\section{DISCUSSION}

Eye closure and loss of lid reflex, however, don't necessarily guarantee a deep enough state of anesthesia to allow for an intense stimulus to be performed. We have found that a common occurrence during sevoflurane induction to overestimate how deeply a child is anesthetized and thus precipitate movement with stimulation. More Movement was noted from 55[4] to $81 \%$ [3] in early group $v s 7$ [4] to $8 \%$ [3] in late groups. In our study, movement was present in group E 12/30 (40\%) vs. no patients in group L. and in secondary outcome measure the difficulty with iv placement. Laryngospasm is defined as the complete or partial loss of airflow through the larynx detected by an inspiratory stridor and passage of air to pulmonary auscultation with movement of the thorax together with movement of the balloon during incomplete laryngospasm and the absence of air passage, respiratory noise and movement of the balloon during complete laryngospasm with no capnogram curve. As soon as the venous approach is successfully applied, a Ringer Lactate infusion is administered. The number of attempts to succeed is recorded for each group. All venous approaches are performed by a trained pediatric anesthesiologist or a senior resident in anesthesia.

\section{RESUlTS}

There were 90 recruited children, 30 in each group. There were no significant demographic differences between age groups $(p=0.485)$, sex and weight $(\mathrm{p}=0.9)$ and ASA $(\mathrm{p}=0.33)$. (Table 1)

Table1: Demographic characteristics patients of group E $v s 27$ others of group L (90s) and 29 cases of group L (120s) with significant difference $(p=0.02)$. At the second attempt, there were 8 cases from group E vs 3 cases group L (90s) and one case group L (120s) $(\mathrm{p}=0.024)$. $($ Table 2$)$
Laryngospasm was recorded in group $\mathrm{E}$, for an incidence of $5.3 \%^{[4]}$ vs $1.3 \%{ }^{[3]}$. In our study, there was no case of laryngospasm in study groups. No significant difference between the groups in terms of difficulty with iv placement: number of attempts in early group is $1.3 \pm 0.7 \mathrm{vs}$ late Group $1.2 \pm 0.7$ [4].

In our study, placement at first attempt was high in group L 90 or $120 \mathrm{~s}$ respectively in 27 and 29 children) vs 22 patients in group E. Our Study concluded that optimum time was between 90 and $120 \mathrm{~s}$. It is recommend waiting for 1 minute 
45 seconds (105 seconds) after the loss of eyelash reflex before attempting intravenous cannulation in pediatric patients induced with sevoflurane, oxygen, and nitrous oxide without any premedication [5]. Schwartz and al [3] justified that for the majority of children, we have shown that it is better to wait 2 min prior to attempting IV placement. Our Study, concluded that optimum time was between $1 \mathrm{~min}$ and half to $2 \mathrm{~min}$ and older children in group $\mathrm{E}$ were more likely to have significant movement.

\section{CONCLUSION}

In conclusion, this study revealed that intravenous cannulation, following an inhalation induction with sevoflurane in children, movement with $i v$ placement was greater when the $i v$ access was attempted $60 \mathrm{~s}$ rather than 90 and $120 \mathrm{~s}$ following loss of lid reflex.

\section{REFERENCES}

[1] Jorge D. Brioni1 · Shane Varughese1 · Raza Ahmed1 - Berthold Bein2 A clinical review of inhalation anesthesia with sevoflurane: from early research to emerging topics $\mathbf{J}$ Anesth (2017) 31:764-778

[2] De Hert S, Moerman A. Sevoflurane. F1000Res. 2015;4:626.

[3] Schwartz d et al. Early intravenous cannulation in children during sevoflurane induction. Pediatr Anaesth2004; 14:820-24.

[4] Choudhry DK, Stayer SA, Schwartz RE et al. Early intravenous cannulation in children during inhalational induction of anaesthesia. Paediatr Anaesth 1998; 8: 123-26.

[5] ABM Kamru, HasanRaman, Sivasankar, Salil G. Nair, Wamia U. Hasan, Zulaidi Latif. Optimum time for intravenous cannulation after induction with sevoflurane, oxygen, and nitrous oxide in children without any premedication . Pediatric Anesthesia December 2017.

Citation: Ben Ali.M, Stambouli L, Hafi F, Ben Salem F, Gahbiche.M. Evaluation of Adequate Time for Intravenous Cannulation in Children during Sevoflurane Induction. ARC Journal of Anesthesiology. 2020; 5(1):01-03. DOI: dx.doi.org/10.20431/2455-9792.0501001.

Copyright: (C) 2020 Authors. This is an open-access article distributed under the terms of the Creative Commons Attribution License, which permits unrestricted use, distribution, and reproduction in any medium, provided the original author and source are credited. 